Förderpreis Pädiatrische

Allergologie

Die Gesellschaft für Pädiatrische Allergologie und Umweltmedizin (GPA) hat den Förderpreis Pädiatrische Allergologie 2004 an den Saarbrückener Qualitätszirkel „Allergologie, Pneumologie und Asthmaschulung im Kindesalter" vergeben. Mit dem von Pharmacia Diagnostics gestifteten Förderpreis werden einmal jährlich Projekte oder Personen gewürdigt, die in besonderer Weise zur Verbesserung der Situation von allergiekranken Kindern und Jugendlichen beigetragen haben.

\section{Allergopharma Award}

Den mit $10.000 €$ dotierten Allergopharma Award 2004 teilen sich Dr. Eleanor Ling, London, und Prof. Dr. Eckard Hamelmann, Berlin. Die Auszeichnung wird seit dem Jahr 2001 in Kooperation mit der European Academy of Allergology and Clinical Immunology (EAACI) an Wissenschaftler vergeben, die durch herausragende Forschungsergebnisse in den Bereichen allergische Entzündung oder spezifische Immuntherapie aufgefallen sind.

\section{Fujisawa-Forschungspreis}

Das Unternehmen Fujisawa hat vergangenes Jahr zum zweiten Mal den Fujisawa-Forschungspreis Immunmodulation an Forscher vergeben, die zu einem besseren Verständnis der Entstehung und Therapie der atopischen Dermatitis beigetragen haben. Den mit $10.000 €$ dotierten Hauptpreis erhielt Prof. Dr. Johannes Norgauer, Jena, die drei mit jeweils $5.000 €$ dotierten Förderpreise gingen an Priv.-Doz. Dr. Axel Trautmann, Würzburg, Priv.-Doz. Dr. Ulf Darsow, München, und an das Forscherteam Priv.-Doz. Dr. Johannes Weiss und Dr. Andreas Renkl, Ulm.

\section{Bayerische Staatsmedaille}

Prof. Dr. Dr. Johannes Ring, Direktor der Klinik und Poliklinik für Dermatologie und Allergologie am Biederstein, Technische Universität München, wurde vom Bayerischen Staatsminister für Umwelt, Gesundheit und Verbraucherschutz, Dr. Werner Schnappauf, mit der Verleihung der „Bayerischen Staatsmedaille für Verdienste um Umwelt und Gesundheit 2004" geehrt.

\title{
Antihistaminika-Verordnung in der Pollensaison 2005
}

\author{
Nach der Verunsicherung der Ärzteschaft durch die 2004 erlassenen \\ Arzneimittelrichtlinien des Gemeinsamen Bundesausschusses \\ sowie die Verlautbarungen von KVen und KBV im letzten Jahr \\ bezüglich der Verordnungsfähigkeit von Antihistaminika sind \\ ähnliche Irritationen auch in diesem Jahr zu Beginn der Saison zu \\ erwarten. Daher nimmt die DGAl wie folgt Stellung.
}

$\mathrm{D}$ ie Unterversorgung von Patienten mit allergischer Rhinitis mit den bekannten Folgen für die Lebensqualität, die Leistungsfähigkeit in Schule und Beruf und die Komorbidität (Asthma-Neuerkrankungen) ist sowohl für die betroffenen Patienten als auch volkswirtschaftlich (indirekte Kosten, Kosten der Folgeerkrankung) nicht zu akzeptieren. Antihistaminika gehören zu den Arzneimitteln der ersten Wahl bei der Behandlung der allergischen Rhinokonjunktivitis.

Bei der Verordnung ist zu beachten: Apothekenpflichtige, nicht verschreibungspflichtige (rezeptfreie) Antihistaminika sind nach der Reform des GMG nur noch in Ausnahmefällen erstattungsfähig. Diese Ausnahmen sind:

— Notfallsets zur Behandlung bei Bienen-, Wespen- oder Hornissengiftallergien,

— schwere, rezidivierende Urtikaria,

— schwerwiegender, anhaltender Pruritus.

Verschreibungspflichtige Antihistaminika dagegen sind unter Berücksichtigung des Wirtschaftlichkeitsgebotes weiterhin erstattungsfähig.

In der Aktualisierung ihrer Stellungnahmen vom 21. April 2004 beziehungsweise vom 15. Juni 2004 stellt die Kassenärztliche Bundesvereinugung den Sachverhalt wie folgt dar:

1. Nicht verschreibungspflichtige Antihistaminika sind in der Indikation „Heuschnupfen“ zu Lasten der gesetzlichen Krankenversicherungen nicht mehr erstattungsfähig.

2. Verschreibungspflichtige Antihistaminika sind - bei Vorliegen der medizinischen Notwendigkeit und Zweckmäßigkeit nach $\$ 12$ SGB V
- unter Berücksichtigung des Wirtschaftlichkeitsgebotes erstattungsfähig.

Der Hinweis auf das Wirtschaftlichkeitsgebot ( $\$ 12$ SGB V) gilt für alle Arzneimittel gleichermaßen, natürlich auch für Antihistaminika. Die Verordnung verschreibungspflichtiger Antihistaminika steht dem Arzt aber unter Beachtung des Wirtschaftlichkeitsgebots frei.

Damit können die Ärzte ihren Patienten weiterhin verschreibungspflichtige Antihistaminika zu Lasten der gesetzlichen Krankenversicherungen verordnen, wenn sie dies für gerechtfertigt und angemessen halten. Im Einzelnen sind dies folgende Gründe, die dokumentiert werden sollten:

- Der Patient ist mit einem verschrei-

bungspflichtigen Antihistaminikum eingestellt und gut therapiert.

- Die mit der Einnahme einiger rezeptfreier Antihistaminika verbundenen Risiken von kardiotoxischen Nebenwirkungen, von Sedierung (Müdigkeit) und psychomotorischen Störungen sowie von Wechselwirkungen mit anderen Arzneimitteln sollen vermieden werden.

_ Der Patient benötigt ein stärker wirksames Antihistaminikum, das insbesondere das Symptom der nasalen Obstruktion (verstopfte Nase) lindert.

\section{Prof. Dr. Claus Bachert, Gent}

